\title{
Genotypic diversity among multidrug resistant Pseudomonas aeruginosa and Acinetobacter species at Mulago Hospital in Kampala, Uganda
}

\author{
David P. Kateete ${ }^{1,2^{*}}$, Ritah Nakanjako ${ }^{1,2}$, Moses Okee ${ }^{1}$, Moses L. Joloba ${ }^{1,2}$ and Christine F. Najjuka ${ }^{2 *}$
}

\begin{abstract}
Background: Multidrug resistant Pseudomonas aeruginosa and Acinetobacter species are common causes of nosocomial infections worldwide. Recently we reported the occurrence of carbapenem resistant Enterobacteriaceae, $P$. aeruginosa and Acinetobacter species at Mulago National Referral Hospital in Kampala, Uganda, but the isolates were not analyzed for genetic relatedness. Herein we report the intra-species genotypic diversity among P. aeruginosa and Acinetobacter baumannii isolated from hospitalized patients and the environment at Mulago Hospital, using repetitive elements-based PCR (Rep-PCR) genotyping.
\end{abstract}

Results: A total of 736 specimens from hospitalized patients were processed for culture and sensitivity testing yielding 9 (1.2\%) P. aeruginosa and 7 (0.95\%) A. baumannii. Similarly, 100 samples from the hospital environment were processed yielding 33 (33\%) P. aeruginosa and 13 (13\%) A. baumannii. Altogether, 62 non-repetitive isolates were studied (42 P. aeruginosa and 20 A. baumannii), of which 38\% (16/42) P. aeruginosa and 40\% (8/20) A. baumannii were multidrug resistant (isolates resistant to three or more classes of antimicrobials). Carbapenem resistance prevalence was 33 and $21 \%$ for P. aeruginosa from patients and the environment, respectively, while it was 14 and $86 \%$ for $A$. baumannii from patients and environment, respectively. Cluster analysis of the Rep-PCR fingerprints revealed a high level of genetic diversity among the isolates within each species as few isolates were clustered (at $100 \%$ level of similarity). More to this, the clustered isolates revealed a complex nature of multidrug resistant $P$. aeruginosa and $A$. baumannii clones circulating at Mulago Hospital. Importantly, certain isolates from the environment and patients were clustered, implying that hospitalized patients at Mulago were probably infected with strains from the environment.

Conclusions: The prevalence of multidrug resistant $P$. aeruginosa and A. baumannii is high at Mulago Hospital but carbapenem resistance prevalence remains relatively low in isolates from hospitalized patients. Importantly, the prevalence of carbapenem resistance in isolates from the environment is high implying the infection control practices at the hospital might be inadequate.

Keywords: Sub-Saharan Africa, Hospital environment, Hospitalized patients, Gram negative bacteria, Antimicrobial resistance AMR, Carbapenems, Genotyping, Rep-PCR

\footnotetext{
*Correspondence: dkateete@chs.mak.ac.ug; najjukafc@gmail.cm

1 Department of Immunology and Molecular Biology, College of Health

Sciences, Makerere University, Kampala, Uganda

2 Department of Medical Microbiology, College of Health Sciences,

Makerere University, Kampala, Uganda
} 


\section{Background}

Pseudomonas aeruginosa and Acinetobacter species are ubiquitous in hospital environments worldwide, and they are frequently implicated among the commonest causes of nosocomial infections that are difficult to treat. This is attributed to the fact that these organisms possess inherent drug resistance mechanisms (e.g. constitutive expression of AmpC beta-lactamases and efflux pumps, low permeability of the outer membrane, etc.), and they are also capable of acquiring additional resistance mechanisms to multiple classes of antimicrobials (e.g. betalactams, aminoglycosides and fluoroquinolones) through horizontal gene transfer [1-3]. Carbapenems have been the most effective drugs used to treat infections caused by multidrug resistant Gram negative bacteria including $P$. aeruginosa and Acinetobacter species. However, the emergence of carbapenemase producing strains, majority of which are multidrug resistant, is threatening the use of carbapenems in the management of such infections $[4,5]$.

Carbapenemase producing $P$. aeruginosa and Acinetobacter were first described in the 1990s and many carbapenemase producing Gram-negative species are increasingly being reported worldwide with increasing rates [4-7]. Of concern, carbapenemase producing Gram negative species appear to be rapidly spreading in sub-Saharan Africa [5, 8-11] pointing to the need of regular surveillance for carbapenem resistant bacteria in hospitals and/or healthcare facilities in low-income settings. Recently, we reported the occurrence of carbapenem resistant Enterobacteriaceae, P. aeruginosa and Acinetobacter baumannii at Mulago National Referral Hospital in Kampala, Uganda [8, 10]. However, the isolates were not analyzed for genetic relatedness. Herein we report the intra-species genotypic diversity among $P$. aeruginosa and A. baumannii isolated from hospitalized patients and the environment at Mulago Hospital, using repetitive elements-based PCR (Rep-PCR) genotyping. Cluster analysis revealed a high level of genetic diversity as few isolates were clustered at $\geq 85 \%$ level of similarity. More to this, the few clustered isolates revealed a complex nature of multidrug resistant $P$. aeruginosa and $A$. baumannii clones circulating at Mulago Hospital.

\section{Results}

Between September 2012 and October 2013, a total of 736 specimens from hospitalized patients were processed for culture and sensitivity testing yielding 9 (1.2\%) P. aeruginosa and 7 (0.95\%) A. baumannii (all non-repetitive isolates). $P$. aeruginosa was isolated from tracheal aspirates (3), urine (2), pus (2), ear swab (1) and sputum (1), while $A$. baumannii was isolated from sputum (3), ear swabs (2), and tracheal aspirates (2). On the other hand, 100 samples from the hospital environment (surgical/ medical wards including the intensive care units, ICUs) that were processed yielded 33 (33\%) P. aeruginosa and 13 (13\%) A. baumannii. Altogether, 62 non-repetitive isolates were characterized (42 P. aeruginosa and $20 \mathrm{~A}$. baumannii).

\section{Antimicrobial susceptibility profiles}

The antimicrobial susceptibility patterns among the isolates are shown in Tables 1 and 2, and Additional file 1: Table S1. None of the isolates from patients were pansusceptible. The overall prevalence of resistance was: amikacin $14 \%$, imipenem (24\%), gentamicin 43\%, ceftazidime $45 \%$, cefepime $7 \%$, aztreonam $48 \%$, piperacillin/tazobactam $62 \%$, ciprofloxacin $48 \%$ for P. aeruginosa; amikacin $15 \%$, gentamicin $35 \%$, meropenem $5 \%$, ceftazidime $30 \%$, cefepime $5 \%$, aztreonam $40 \%$, piperacillin/ tazobactam $70 \%$, ciprofloxacin $40 \%$, and trimethoprim/ sulfamethoxazole (SXT) $60 \%$ for $A$. baumannii. All $P$. aeruginosa isolates were susceptible to meropenem while

Table 1 Antimicrobial resistance rates among $P$. aeruginosa (\% resistant)

\begin{tabular}{|c|c|c|c|}
\hline Antimicrobial & Hospitalized patients $n=9$ & Environment $\mathrm{n}=33$ & Total $\mathrm{N}=42$ \\
\hline \multicolumn{4}{|l|}{ Beta-lactam agents } \\
\hline Aztreonam & $4(44 \%)$ & $16(48 \%)$ & $20(48 \%)$ \\
\hline Cefepime & $3(33 \%)$ & 0 & $3(7 \%)$ \\
\hline Ceftazidime & $5(56 \%)$ & $14(42 \%)$ & $19(45 \%)$ \\
\hline Imipenem & $3(33 \%)$ & $7(21 \%)$ & $10(24 \%)$ \\
\hline Meropenem & 0 & 0 & 0 \\
\hline Piperacillin-tazobactam & $5(56 \%)$ & $21(64 \%)$ & $26(62 \%)$ \\
\hline \multicolumn{4}{|l|}{ Aminoglycosides } \\
\hline Amikacin & $2(22 \%)$ & $4(12 \%)$ & $6(14 \%)$ \\
\hline Gentamicin & $5(56 \%)$ & $13(39 \%)$ & $18(43 \%)$ \\
\hline \multicolumn{4}{|l|}{ Quinolones } \\
\hline Ciprofloxacin & $7(78 \%)$ & 13 (39\%) & 20 (48\%) \\
\hline
\end{tabular}


Table 2 Antimicrobial resistance rates among A. baumannii (\% resistant)

\begin{tabular}{|c|c|c|c|}
\hline Antimicrobial & Hospitalized patients $n=7$ & Environment $\mathrm{n}=13$ & Total $\mathrm{N}=20$ \\
\hline \multicolumn{4}{|l|}{ Beta-lactam agents } \\
\hline Aztreonam & $3(43 \%)$ & $5(34 \%)$ & $8(40 \%)$ \\
\hline Cefepime & $1(14 \%)$ & 0 & $1(5 \%)$ \\
\hline Ceftazidime & $5(71 \%)$ & $1(8 \%)$ & $6(30 \%)$ \\
\hline Imipenem & $1(14 \%)$ & $6(86 \%)$ & $7(35 \%)$ \\
\hline Meropenem & $1(14 \%)$ & 0 & $1(5 \%)$ \\
\hline Piperacillin/tazobactam & $3(43 \%)$ & $11(85 \%)$ & $14(70 \%)$ \\
\hline \multicolumn{4}{|l|}{ Aminoglycosides } \\
\hline Amikacin & $3(43 \%)$ & 0 & $3(15 \%)$ \\
\hline Gentamicin & $5(71 \%)$ & $2(15 \%)$ & $7(35 \%)$ \\
\hline \multicolumn{4}{|l|}{ Quinolones } \\
\hline Ciprofloxacin & $6(86 \%)$ & $2(15 \%)$ & $8(40 \%)$ \\
\hline \multicolumn{4}{|l|}{ Others } \\
\hline Trimethoprim/sulfamethoxazole (SXT) & $4(57 \%)$ & $8(62 \%)$ & $12(60 \%)$ \\
\hline
\end{tabular}

three isolates from patients and seven from the environment were imipenem resistant, Table 1.

Overall, carbapenem resistance prevalence was 33 and $21 \%$ for $P$. aeruginosa from patients and the environment, respectively, while it was 14 and $86 \%$ for $A$. baumannii from patients and environment, respectively. Furthermore, all the imipenem resistant $P$. aeruginosa isolates were also resistant to aztreonam while $90 \%$ were resistant to piperacillin/tazobactam. On the other hand, all carbapenem resistant $A$. baumannii were resistant to piperacillin/tazobactam while $57 \%$ were aztreonam resistant. Of note, SXT-susceptible, carbapenem resistant $A$. baumannii from patients and the environment was noted, Table 2 and Additional file 1: Tables S1, Additional file 2: Table S2. Furthermore, 38\% (16/42) of P. aeruginosa and $40 \%(8 / 20)$ of $A$. baumannii isolates were multidrug resistant (resistance to three or more antimicrobial classes [12]).

\section{Beta-lactamases}

Overall, $67 \%$ of the carbapenem resistant isolates were positive with the imipenem/EDTA double disk synergy test, implying the isolates were metallo-beta-lactamase producers, Additional file 1: Table S1. Hence, metallo-beta-lactamases were responsible for carbapenem resistance in majority of the carbapenem resistant isolates studied. Furthermore, 60\% (37/62) of the isolates were resistant either to aztreonam or ceftazidime (extended spectrum cephalosporins), implying they were suspects for AmpC (class C $\beta$-lactamase enzyme/cephalosporinase) and extended spectrum beta-lactamases (ESBLs) production. However, none of these isolates was positive on ESBL and AmpC screening.

\section{Genotypic diversity}

Genotypic diversity within each species was investigated using Rep-PCR genotyping. Rep-PCRs produced 0-15 consistent polymorphic bands per isolate of sizes 2509400 bp (BOXAIR-PCR), 90-8000 bp (ERIC-PCR), and 100-6557 bp (REP-PCR), Additional file 3: Figure S1.

At $100 \%$ similarity scale, BOXAIR-PCR fingerprints for A. baumannii isolates were not clustered (Fig. 1); more to this for Acinetobacter, REP-PCR fingerprints produced only one cluster (Fig. 2) while ERIC-PCR fingerprints produced two clusters (Additional file 4: Figure S2). Isolates 59 and 62 (both carbapenem susceptible) from a patient and environment, respectively, were considered genetically related in that their fingerprints were clustered at $100 \%$ similarity scale by both ERIC-PCR and REP-PCR cluster analysis, Fig. 2 and Additional file 4: Figure S2. Altogether, only five Acinetobacter isolates were clustered at $100 \%$ similarity scale. This implies low level of genetic relatedness among the isolates. More to this, the few clustered isolates also reveal a complex nature of $A$. baumannii multidrug resistant clones circulating at Mulago Hospital in that the isolates exhibited diverse antimicrobial susceptibility phenotypes, Additional file 2: Table S2.

For $P$. aeruginosa, cluster analysis at $100 \%$ similarity scale with BOXAIR-PCR, ERIC-PCR, and REP-PCR fingerprints generated one, two and three clusters, respectively, Fig. 3 and Additional file 5: Figures S3, Additional file 6: Figure S4. Isolates 20 and 21 from the environment were considered genetically related as their fingerprints were clustered by both BOXAIR-PCR and REP-PCR analysis, Fig. 3 and Additional file 6: Figure S4. As observed above for Acinetobacter, isolates from a patient and environment 


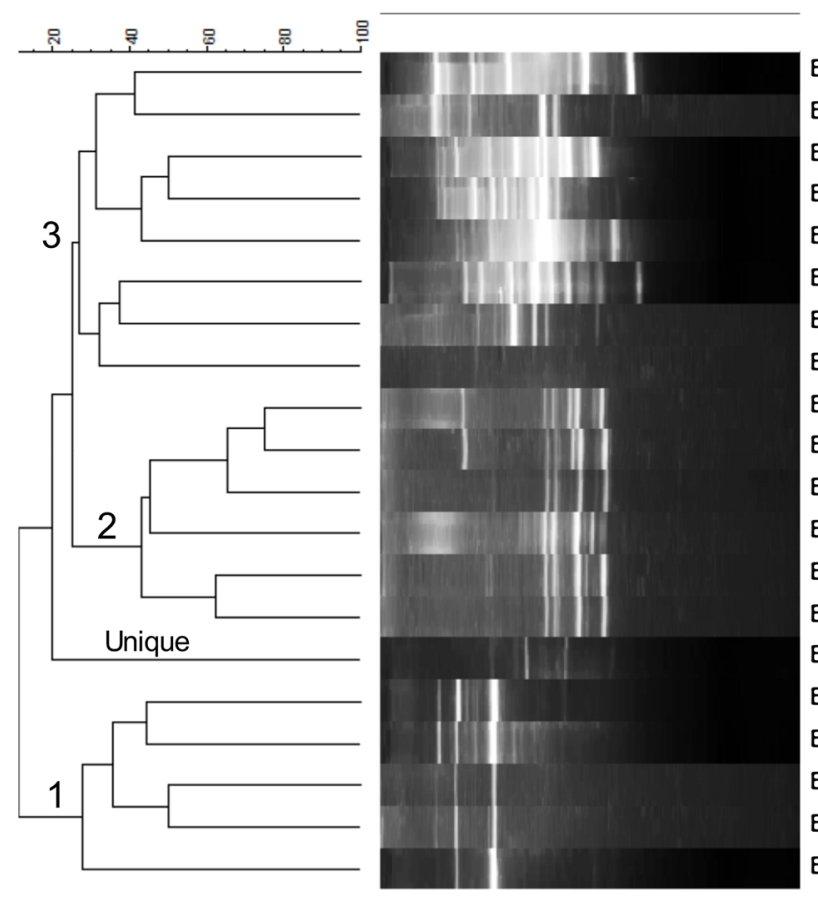

\begin{tabular}{|c|c|}
\hline & Susceptibility profiles \\
\hline Bi50A.baumanii (E) & SXT-TZP-IMP \\
\hline Bi54A.baumanii (E) & SXT-TZP \\
\hline Bi48A.baumanii (E) & - \\
\hline Bi49A. baumanii $(E)$ & TZP-IMP-ATM \\
\hline Bi51A.baumanii (E) & CIP-SXT-TZP-IMP-ATM ${ }^{b}$ \\
\hline Bi46A.baumanii (E) & CN-TZP-IMP-ATM ${ }^{c}$ \\
\hline Bi55A.baumanii $(E)$ & TZP-IMPc \\
\hline Bi52A.baumanii $(E)$ & CIP-SXT-CAZ-TZP-ATM-IMP' \\
\hline Bi58A.baumanii $(H)$ & CIP-SXT-CAZ \\
\hline Bi61A.baumanii $(E)$ & CN-CAZ-CIP-SXT' \\
\hline Bi62A.baumanii (E) & $\mathrm{CN}-\mathrm{CAZ}$ \\
\hline Bi57A.baumanii $(H)$ & CIP-CN-AK-ATM-TZP' \\
\hline Bi59A baumanii $(H)$ & CIP-CN-TZP-CAZ-FEP-AK-ATM-SXT' \\
\hline Bi60A.baumanii $(\mathrm{H})$ & CIP-CN-AK-SXT-CAZ-ATM ${ }^{\mathbf{b}}$ \\
\hline Bi43A. baumanii $(H)$ & CIP-TZP-IMP-MEM' \\
\hline Bi44A.baumanii (E) & CIP-TZP-SXT-ATM ${ }^{b}$ \\
\hline Bi47A baumanii $(E)$ & TZP-SXT \\
\hline Bi53A.baumanii (E) & TZP-SXT \\
\hline Bi56A.baumanii (E) & TZP \\
\hline Bi45A.baumanii (E) & SXT-CN \\
\hline
\end{tabular}

Fig. 1 Dendrogram based on Dice's coefficient of similarity using UPGMA method implemented by the Bionumerix program showing relationships between $A$. baumannii isolates according to BOXAIR-PCR genotyping. $E$ isolate from hospital environment; $H$ isolate from hospitalized patient. The susceptibility profiles (extreme right panel) for carbapenem-resistant isolates are indicated in brown font. AK amikacin, CN gentamicin, IMP imipenem, MEM meropenem, CAZ ceftazidime, FEP cefepime, ATM aztreonam, TZP piperacillin/tazobactam, CIP ciprofloxacin, SXT trimethoprim/ sulfamethoxazole. ${ }^{b}$ Multidrug-resistance pattern; ${ }^{C}$ SXT-susceptible carbapenem-resistant A. baumannii

(1 and 25, respectively) were also clustered by ERIC-PCR analysis, Additional file 5: Figure S3. Likewise for P. aeruginosa, few isolates (10 of 42, 24\%) generated fingerprints that were clustered at $100 \%$ similarity scale, implying a low level of genetic relatedness but a complex nature of multidrug resistant $P$. aeruginosa clones could be circulating at Mulago Hospital given the diverse antimicrobial susceptibility profiles generated by the clustering isolates, Additional file 2: Table S2.

The Simpson's index of discrimination (D) for P. aeruginosa fingerprints was 0.9829 (BOXAIR-PCR), 0.9756 (ERIC-PCR), and 0.9791 (REP-PCR), indicating that the three genotyping methods successfully discriminated $P$. aeruginosa but BOXAIR-PCR and REP-PCR performed better than ERIC-PCR. For A. baumannii, Simpson's index of discrimination was 0.9632 (BOXAIR-PCR), 0.9608 (ERIC-PCR), and 0.9474 (REP-PCR) indicating that the three methods also successfully discriminated the isolates but BOXAIR-PCR and ERIC-PCR performed better.

\section{Discussion}

In this study, we found the prevalence of multidrug resistant $P$. aeruginosa and $A$. baumannii at Mulago Hospital to be high but comparable to rates reported by Pitout et al. in Kenya [11], Lee et al. in Korea [13] and $\mathrm{Gu}$ et al. in China [14]. Additionally, the rates in the current study are lower than those reported by Uma Karthika et al. in India [15]. This could reflect challenges in embracing antibiotic stewardship programmes, or the differences in the amounts of antibiotics consumed in each setting where those studies were conducted [16]. Importantly, in Uganda like many other lowincome countries, antibiotics are readily available over the counter in community pharmacies $[9,17]$ and this portends high rates of antimicrobial resistance in lowincome countries.

Furthermore, carbapenem resistance prevalence in this study remains low in isolates from patients in accordance with recent findings at Mulago $[8,10,18]$, and significantly lower than rates from Latin America [19]. Yet, carbapenem resistance in this study was high in isolates from the environment, possibly due to the inadequate infection control practices at the hospital [8]. Notably, carbapenem resistant Acinetobacter species that were SXT-susceptible were noted. Generally, SXT is not very active against Acinetobacter but it was recommended where there are no other options [20], e.g. for infections with pan-resistant strains or in settings where efficacious drugs like carbapenems are expensive [18]. 

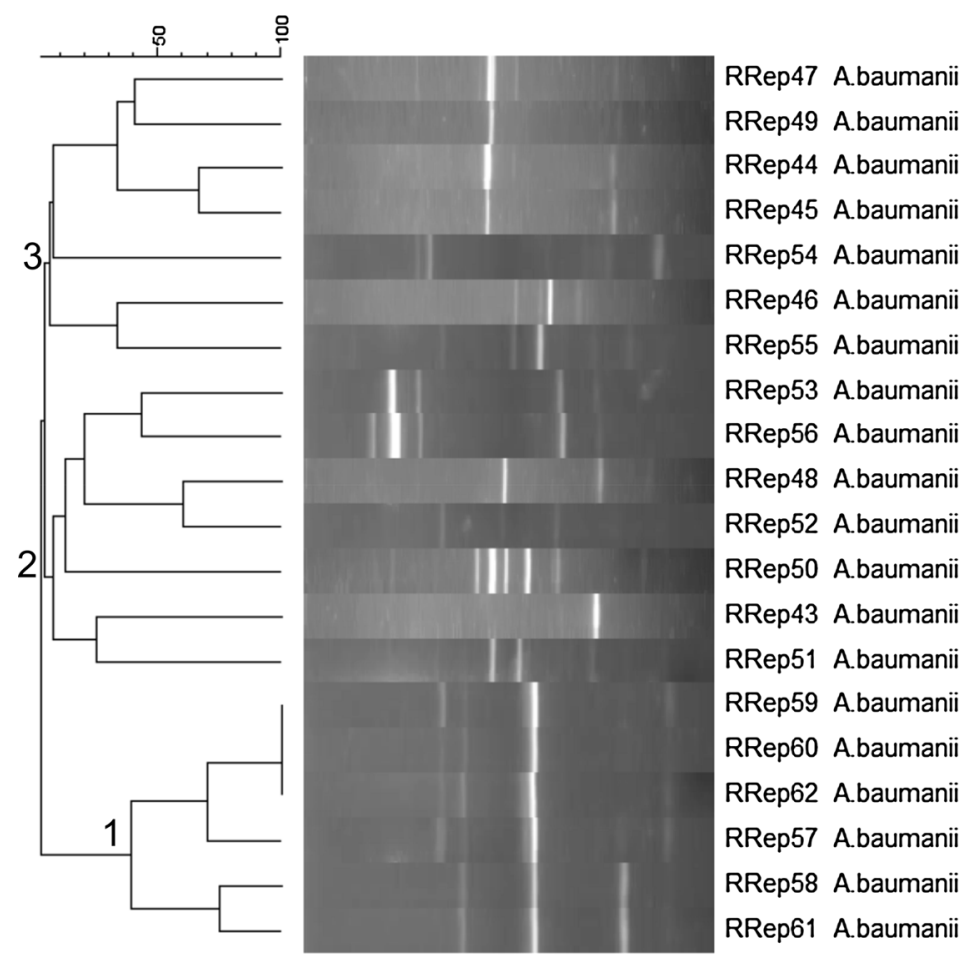

\begin{tabular}{|c|c|}
\hline & Susceptibility profiles \\
\hline (E) & TZP-SXT \\
\hline (E) & TZP-IMP-ATM" \\
\hline (E) & CIP-TZP-SXT-ATM ${ }^{B}$ \\
\hline (E) & SXT-CN \\
\hline$(E)$ & TZP-SXT \\
\hline (E) & CN-TZP-IMP-MEMC \\
\hline (E) & IMP-TZPc \\
\hline (E) & TZP-SXT \\
\hline (E) & TZP \\
\hline (E) & - \\
\hline (E) & CIP-SXT-CAZ-TZP-ATM-IMP' \\
\hline (E) & SXT-TZP-IMP \\
\hline$(\mathrm{H})$ & CIP-TZP-IMP-MEM ${ }^{c}$ \\
\hline$(\mathrm{H})$ & CIP-SXT-TZP-IMP-ATM b \\
\hline (H) & CIP-CN-TZP-CAZ-FEP-AK-ATM-SXT' \\
\hline$(H)$ & CIP-CN-AK-SXT-CAZ-ATM ${ }^{b}$ \\
\hline (E) & $\mathrm{CN}-\mathrm{CAZ}$ \\
\hline$(H)$ & CIP-CN-AK-ATM-TZP \\
\hline$(\mathrm{H})$ & CIP-SXT-CAZ \\
\hline (E) & CN-CAZ-CIP-SXT' \\
\hline
\end{tabular}

Fig. 2 Dendrogram based on Dice's coefficient of similarity using UPGMA method implemented by the Bionumerix program showing relationships between $A$. baumannii isolates according to REP-PCR genotyping. $E$ isolate from hospital environment; $H$ isolate from hospitalized patient. The susceptibility profiles (extreme right panel) for carbapenem-resistant isolates are indicated in brown font. AK amikacin, CN gentamicin, IMP imipenem, MEM meropenem, CAZ ceftazidime, FEP cefepime, ATM aztreonam, TZP piperacillin/tazobactam, CIP ciprofloxacin, SXT trimethoprim/sulfamethoxa-

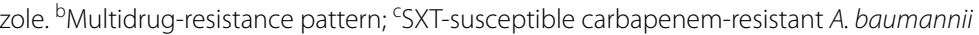

Although the metallo-beta-lactamases were associated with imipenem resistance in this study, it is important to note that carbapenemases are not the only mechanisms of acquired resistance to carbapenems [21]. Other mechanisms that were not investigated in this study, such as loss or reduced expression of the outer membrane porin OprD, are associated with imipenem resistance; likewise, upregulation of efflux pumps (e.g. MexAB-OprM) combined with OprD loss is associated with meropenem resistance.

Additionally, it is widely accepted that aztreonam susceptibility combined with resistance to carbapenems and other beta-lactams is a "classic" metallo-beta-lactamase phenotype [21]. However, metallo-beta-lactamase producing strains that are resistant to aztreonam do occur, implying that the "classic" metallo-beta-lactamase pattern is not always seen [21]. Indeed in this study, the prevalence of aztreonam resistance was high among carbapenem resistant isolates. Further, although metallo-beta-lactamase producing strains do not generally hydrolyze monobactams (e.g. aztreonam), metallo-betalactamase producing $P$. aeruginosa strains that are resistant to aztreonam have been reported. This is not surprising in that production of a metallo-beta-lactamase does not exclude presence of intrinsic resistance mechanisms in $P$. aeruginosa, e.g. up-regulated efflux pumps or outer membrane impermeability [21] outlined above. Indeed, carbapenem resistant $P$. aeruginosa and Acinetobacter strains that are resistant to aztreonam have been reported by researchers worldwide [22]. Taken together, there is surprisingly high level of aztreonam resistance in $P$. aeruginosa isolated from hospital environments [23]. In a study by Santoro et al. [24] on isolates from the hospital wastewater treatment system and clinical specimens in a hospital in Rio de Janeiro city Brazil, $63 \%$ of $P$. aeruginosa isolates from sewage exhibited decreased susceptibility to aztreonam while $50 \%$ of the clinical isolates were resistant to this drug [24].

In a recent study at Mulago National Referral Hospital, more than three quarters of the Enterobacteriaceae were found to be ESBL-producers [18]. Although resistance to extended-spectrum cephalosporins (aztreonam and ceftazidime) was high in this study, ESBLs and AmpC activity was not detected implying that the genes encoding these enzymes might have not yet spread to $P$. aeruginosa and Acinetobacter strains at Mulago. However, we could have missed detecting ESBLs and AmpC 


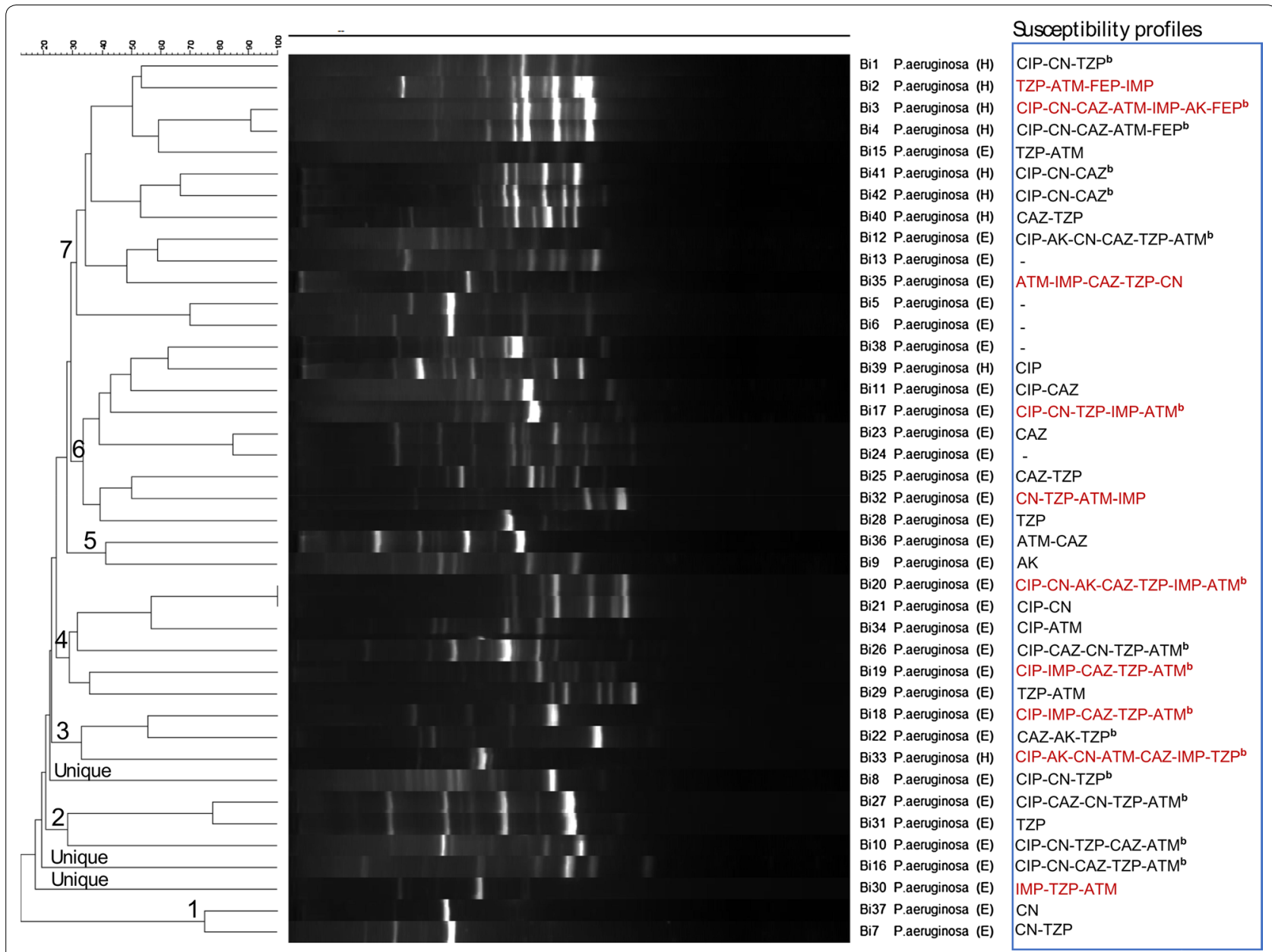

Fig. 3 Dendrogram based on Dice's coefficient of similarity using the UPGMA method implemented by the Bionumerix program showing relationships between $P$. aeruginosa isolates according to BOXAIR-PCR genotyping. $E$ isolate from hospital environment; $H$ isolate from hospitalized patient. The susceptibility profiles (extreme right panel) for carbapenem-resistant isolates are indicated in brown font. AK Amikacin, CN gentamicin, IMP imipenem, MEM meropenem, CAZ ceftazidime, FEP cefepime, ATM aztreonam, TZP piperacillin/tazobactam, CIP ciprofloxacin. ${ }^{b}$ Multidrug-resistance pattern

producing isolates as the double disc synergy test we used is not very efficient at detecting these enzymes particularly when ESBL activity is low, leading to wide zones of inhibition around cephalosporin/aztreonam discs [25]. As the $A m p C$ and ESBL genes are transmissible via horizontal gene transfer mechanisms and are associated with multidrug resistance, AmpC and ESBL screening in organisms other than Escherichia coli, Klebsiella pneumoniae, Klebsiella oxytoca, and Proteus mirabilis in hospitals where ESBLs and AmpC are encountered is considered an important component of infection control programmes [26]. Therefore, continual surveillance at Mulago for AmpC/ESBL-producing P. aeruginosa and Acinetobacter species is necessary.

Generally, cluster analysis in this study revealed a low level of genetic relatedness among the isolates. The few clustered isolates point to likely occurrence of complex clones of multidrug resistant $P$. aeruginosa and $A$. baumannii circulating at Mulago Hospital as the isolates exhibited diverse antimicrobial susceptibility patterns. Further studies with a larger number of isolates are necessary to obtain a better picture of the circulating clones at Mulago Hospital. Other researchers who used RepPCR genotyping also reported a high level of genetic diversity among $P$. aeruginosa isolated from a hospital in Belo Horizonte, Brazil [22]. Moreover, a nonclonal epidemic population structure of $P$. aeruginosa, supported by lack of evidence for a widespread cystic fibrosis transmissible clone, has been reported [27].

On the other hand, the high genetic diversity among isolates in this study might also point to either a high host diversity from where the bacteria were isolated, or lack of infection control practices. As the only national referral hospital in Uganda, Mulago admits patients from the 
whole country characterized by variation in geographic landscapes, human populations and human development index (HDI) [9]. Although P. aeruginosa and Acinetobacter species colonizing hospital settings in high income countries are characterized with clonality, the picture might be different in low-income settings characterized with overcrowding of patients, lack of adequate infection control and proper hygiene. Indeed, hospitals in Uganda are overcrowded with clients from all over the country hence, maintaining environmental hygiene becomes tedious [28]. More to this, the 'my five moments for hand hygiene' designed for healthcare environments describes recommended levels of bed spacing and occupancy that are normally feasible in high-income countries. Overcrowding of healthcare facilities in low-income countries is often worsened by minimal infection control practices as evidenced by low standards of hand hygiene among health care workers [28]. With low standards of environmental hygiene, bacteria like Pseudomonas are likely to persist in hospital environments where they easily exchange/acquire resistance genetic elements. There is also uncontrolled use of drugs like the third generation cephalosporins in low-income settings and this facilitates selection of diverse drug resistant strains within the healthcare facility [29].

\section{Conclusions}

The prevalence of multidrug resistant $P$. aeruginosa and A. baumannii is high at Mulago Hospital. Carbapenem resistance prevalence remains low in isolates from hospitalized patients but high in the environment, possibly due to inadequate infection control practices. As isolates from patients and environment were clustered, hospitalized patients are at high risk of infection with multidrug resistant strains from the environment. Although the repetitive sequence elements on which Rep-PCR genotyping is based are highly conserved throughout the prokaryotic kingdom [30] and have been used extensively in the surveillance of $P$. aeruginosa and Acinetobacter species and other bacterial species [31-35], we recommend further studies employing modern genotyping methods, e.g. pulse field gel electrophoresis and/or DNA sequencing-based approaches (the gold standard for bacterial typing) that were not affordable in the current study.

\section{Methods \\ Setting}

This was a follow-up surveillance study conducted at Mulago Hospital in Kampala Uganda, following the detection of carbapenem resistant Gram negative bacteria at the hospital in the period 2007-2009 [8, 10]. A total of 736 specimens from hospitalized patients were referred to the Clinical Microbiology and Molecular Diagnostics Laboratories at the College of Health Sciences, Makerere University, for culture and sensitivity testing. Additionally, 100 samples from the hospital environment [water, disinfectants in use (chlorhexidine gluconate), wet sink swabs, wet floors swabs, and swabs of cleaning materials (mops, squeezers)] were randomly collected and similarly processed for isolation of Pseudomonas and Acinetobacter species.

\section{Identification of $P$. aeruginos $a$ and Acinetobacter species}

The sample collection and processing procedure was described in Kateete et al. [8]. Briefly, samples/specimens were processed within $2 \mathrm{~h}$ of collection for identification of Gram negative bacteria by following standard microbiological/biochemical procedures [8]. The recovered isolates were first presumptively identified based on colony morphology, Gram-staining properties and standard biochemical characteristics. Characteristic colony morphological features (i.e. colonies with characteristic spreading pattern and serrated edges, fruity sweet-grape smell, and bright green color) were used to identify Pseudomonas. Positive catalase and oxidase tests, negative triple sugar iron and glucose fermentation tests and growth at $42{ }^{\circ} \mathrm{C}$ were used to distinguish $P$. aeruginosa from other lactose non-fermenting Gram-negative rods. Acinetobacter species were presumptively identified based on negative motility, catalase, oxidase and glucose fermentation tests, and inability to grow under aerobic conditions.

To confirm Acinetobacter isolates to species level, the 'Phoenix ${ }^{\mathrm{TM}}$ Automated Microbiology System' (Becton and Dickson, Franklin Lakes, NJ, USA) was used; in addition, PCR-amplification followed by DNA sequencing of the species-specific region of the $b l a_{\text {OXA-51 }}$ gene intrinsic to A. baumannii $[5,36]$ was performed. To confirm $P$. aeruginosa to species level, and to determine the antimicrobial susceptibility profiles of both $A$. baumannii and $P$. aeruginosa isolates, minimum inhibitory concentrations (MICs) were performed with the 'Phoenix ${ }^{\mathrm{TM}}$ Automated Microbiology System' according to the manufacturer's guidelines with minor modifications for Gram negative bacteria as described elsewhere [8, 37]. In addition, the disc diffusion method was performed to determine SXT susceptibility of $A$. baumannii isolates, according to the Clinical and Laboratory Standards Institute (CLSI) guidelines. Quality control and maintenance for the 'Phoenix ${ }^{\mathrm{TM}}$ Automated Microbiology System' were performed as recommended by the manufacturer using reference strains of E. coli ATCC 25922 and P. aeruginosa ATCC 27853. Phoenix $^{\text {TM }}$ default MIC-breakpoints were described in Kateete et al. [8]. 


\section{Metallo- $\beta$-lactamase assays}

All the carbapenem-resistant isolates were tested for metallo- $\beta$-lactamase activity as bacteria producing these enzymes are characterized by rapid spread worldwide [5]. Assays were performed with the imipenem-EDTA (Ethylenediaminetetraacetic acid) double-disk synergy test using K. pneumoniae ATCC 700603 and E. coli ATCC 25922 as indicator strains $[10,38,39]$. An overnight liquid culture of the test isolate was adjusted to a turbidity of 0.5 McFarland standard and spread on the surface of a Mueller-Hinton Agar (MHA) plate. Two imipenem discs $(10 \mu$ g each) were placed on the agar $15 \mathrm{~mm}$ apart (center-to-center); $10 \mu \mathrm{l}$ of $0.5 \mathrm{M}$ EDTA was added to one of the imipenem disc to get a desired concentration of $750 \mu \mathrm{g}$. After incubating at $37^{\circ} \mathrm{C}$ overnight, the difference in the inhibition zone diameter that was $\geq 5 \mathrm{~mm}$ between the imipenem disc supplemented with EDTA and imipenem disc alone was interpreted as positive for metallo- $\beta$-lactamase production [21, 39]. K. pneumoniae strains ATCC BAA-1705 and ATCC BAA-1706 served as positive and negative controls, respectively; strain BAA1705 possesses a $K$. pneumoniae carbapenemase KPC-2 that is highly active against cephamycins, carbapenems, and to a substrate range of ESBLs [40]. Positive or negative results were interpreted according to the CLSI guidelines and the UK Standards for Microbiology Investigations $[21,39,41]$.

\section{ESBL and AmpC screening}

Isolates that exhibited resistance to extended-spectrum cephalosporins (ceftazidime and aztreonam) were subjected to the double disk synergy test to detect production of ESBLs as described elsewhere [26], by placing ceftazidime, cefotaxime, aztreonam and ceftriaxone discs (30 $\mu \mathrm{g}$ each) at $20 \mathrm{~mm}$ distance (center-to-center) from amoxicillin $(20 \mu \mathrm{g})$ and clavulanic acid $(10 \mu \mathrm{g})$ discs. ESBL activity was inferred if the cephalosporin zone was expanded by the clavulanic acid containing disk. Ceftazidime and/or aztreonam resistant isolates were also tested for production of plasmid-mediated $\mathrm{AmpC}$ as previously described [26] using MHA plates and ceftazidime $(30 \mu \mathrm{g})$ and cefoxitin $(30 \mu \mathrm{g})$ discs placed $20 \mathrm{~mm}$ (center-tocenter). AmpC-production was considered when isolates showed blunting of ceftazidime zone of inhibition adjacent to cefoxitin disc.

\section{Genotyping, electrophoresis and analysis of DNA fingerprints}

Genotyping was performed with Rep-PCR assays (REP-, BOXAIR- and ERIC-PCRs). Initially, we followed the procedure described by Syrmis et al. [35] to optimize the concentration of $\mathrm{Mg}++$, template DNA, Taq polymerase and PCR primers, using four unrelated isolates of $P$. aeruginosa and $A$. baumannii. Chromosomal DNA used as templates in PCRs was extracted by the Cetyltrimethyl Ammonium Bromide (CTAB) method [42, 43] and dissolved in $100 \mu \mathrm{l}$ of sterile Tris-EDTA (TE) buffer. $100 \mathrm{ng}$ of the extracted DNA was used as template in the PCRs. REP-, BOXAIR- and ERIC-PCRs were performed using previously described primers and conditions [32, 44], with minor modifications to suit our setting. Ten microliters each of the PCR products was size-fractionated by agarose gel electrophoresis $(1.5 \% \mathrm{w} / \mathrm{v}$ agarose) at constant voltage of $40 \mathrm{~V}$ in $1 \times$ TAE buffer (Tris-acetate $\mathrm{pH}$ 8.3, $40 \mathrm{mM}$ Tris, $20 \mathrm{mM}$ acetic acid, $1 \mathrm{mM}$ EDTA) for $8 \mathrm{~h}$ in a horizontal electrophoretic apparatus (Bio-Rad Inc.). For quality control and uniformity in DNA migration during electrophoresis, all the gels were electrophoresed for equal amount of time. DNA size markers contained a mixture of the 50 base pairs (bp) DNA ladder (50-1350 bp size range) plus $\lambda$ DNA-HindIII digest DNA ladder (2027-23,130 bp visible size range) (New England Biolabs Inc.). REP-, ERIC-, and BOXAIR-PCR profiles were visualized under ultraviolet (UV) light after staining the gels with ethidium bromide for $30 \mathrm{~min}$. Images were captured and stored as TIFF files using BioDoc-it Imaging system (UVP, Cambridge, UK).

DNA fingerprints were analyzed with the BioNumerics software version 4 (Applied Maths NV, Sint-MartensLatem, Belgium) as described elsewhere [34, 35, 45, 46]. The similarity-analysis of the results of each genotyping assay was calculated using Dice coefficient; cluster analysis of the similarity matrices was generated using Unweighted Pair Group Method with Arithmetic Mean (UPGMA) algorithm. The relationship between profiles was expressed as dendrograms; only visible bands were used to construct the similarity matrices and dendrograms. Variation in band intensity was not considered as genetic difference. Isolates were considered identical when all the scored bands in each pattern had similar migration patterns/distance. As few isolates shared similar migration patterns, the criterion for relatedness was taken as profiles with $\geq 85 \%$ similar migration patterns/ distance [35]. The Simpson's index of diversity [47, 48] was used to estimate discrimination indices ( $D$ values) for the three typing methods.

\section{Quality control}

Controls for Rep-PCR genotyping included known well characterized strains K. pneumoniae DSMZ 9377, E. coli ATCC 25922 and P. aeruginosa ATCC 27853. We optimized Rep-PCR assays using the procedure described for Yersinia [46] and adapted it to P. aeruginosa and $A$. baumannii. Genotyping was performed at least twice in separate assays to ensure reproducibility of the patterns; BOXAIR-PCR, ERIC-PCR, and REP-PCRs were 
repeated for five unrelated isolates of $P$. aeruginosa and A. baumannii. PCRs and electrophoresis were performed in two separate trials starting from the same DNA preparation, using the same PCR reagents. None of the isolates showed qualitative differences in the fingerprints. We also investigated the effect of the DNA preparation method using chromosomal DNA extracted with two inhouse methods of CTAB and boiling; although CTABprepared DNA consistently yielded fingerprints with stronger band intensities, no significant difference in band sizes was detected suggesting stability of our RepPCR fingerprints.

\section{Additional files}

Additional file 1: Table S1. Antimicrobial resistance profiles of P. aeruginosa and A. baumannii.

Additional file 2: Table S2. Antimicrobial resistance profiles of isolates that were clustered at $100 \%$ similarity scale. The diverse antimicrobial susceptibility profiles exhibited by the clustered isolates reveal a complex nature of multidrug resistant $P$. aeruginosa and $A$. baumannii clones circulating at Mulago Hospital.

Additional file 3: Figure S1. Representative image showing agarose gel electrophoresis of $P$. aeruginosa and $A$. baumannii PCR fingerprints following BOXAIR-PCR genotyping. Panels A and B, lanes 18-42, P. aeruginosa fingerprints; panel $B$, lanes 43-51, A. baumannii fingerprints; lane $M$, DNA size marker that was reconstituted by mixing 50 base pair-DNA ladder (50$1350 \mathrm{bp}$ size range) with $\lambda$ DNA-Hindlll digest-DNA ladder (2027-23,130 bp visible size range). Carbapenem-resistant isolates are indicated in red font.

Additional file 4: Figure S2. Dendrogram based on Dice's coefficient of similarity using the UPGMA method implemented by the Bionumerix program showing relationships between A. baumannii isolates according to ERIC-PCR genotyping. (E), isolate from hospital environment; $(\mathrm{H})$ isolate from hospitalized patient. The susceptibility profiles (extreme right panel) for carbapenem-resistant isolates are indicated in brown font; AK, Amikacin; CN, Gentamicin; IMP, Imipenem; MEM, Meropenem; CAZ, Ceftazidime; FEP, Cefepime; ATM, Aztreonam; TZP, Piperacillin/tazobactam; CIP, Ciprofloxacin; SXT, Trimethoprim/sulfamethoxazole. ${ }^{b}$ Multidrug-resistance pattern; 'SXT-susceptible carbapenem-resistant $A$. baumannii.

Additional file 5: Figure S3. Dendrogram based on Dice's coefficient of similarity using the UPGMA method implemented by the Bionumerix program showing relationships between $P$. aeruginosa isolates according to ERIC-PCR genotyping. (E), isolate from hospital environment; $(\mathrm{H})$, isolate from hospitalized patient. The susceptibility profiles (extreme right panel) for carbapenem-resistant isolates are indicated in brown font: AK, Amikacin; CN, Gentamicin; IMP, Imipenem; MEM, Meropenem; CAZ, Ceftazidime; FEP, Cefepime; ATM, Aztreonam; TZP, Piperacillin/tazobactam; CIP, Ciprofloxacin. ${ }^{b}$ Multidrug-resistance pattern.

Additional file 6: Figure S4. Dendrogram based on Dice's coefficient of similarity using the UPGMA method implemented by the Bionumerix program showing relationships between $P$. aeruginosa isolates according to REP-PCR genotyping. (E), isolate from hospital environment; $(H)$, isolate from hospitalized patient. The susceptibility profiles (extreme right panel) for carbapenem-resistant isolates are indicated in brown font; AK, Amikacin; CN, Gentamicin; IMP, Imipenem; MEM, Meropenem; CAZ, Ceftazidime; FEP, Cefepime; ATM, Aztreonam; TZP, Piperacillin/tazobactam; $\mathrm{CIP}$, Ciprofloxacin. ${ }^{\mathrm{b}}$ Multidrug-resistance pattern.

\section{Abbreviations}

ICU: intensive care unit; MICs: minimum inhibitory concentrations; SXT: trimethoprim/sulfamethoxazole; CLSI: Clinical and Laboratory Standards
Institute; EDTA: ethylenediaminetetraacetic acid; KPC: Klebsiella pneumoniae carbapenemase; ESBLs: extended spectrum beta-lactamases; Rep-PCR: repetitive elements-based PCR; TE: Tris-EDTA; TAE: tris acetic acid EDTA buffer; UPGMA: Unweighted Pair Group Method with Arithmetic Mean; UV: ultraviolet.

\section{Authors' contributions}

DPK and CFN conceived and designed the study; they also supervised the molecular assays, performed data analysis and drafted the manuscript. RN performed the microbiology and molecular assays in partial fulfilment of the requirements for the award of the Degree of Master of Science in Molecular Biology of Makerere University (under supervision of CFN and DPK). MO analyzed the Rep-PCR fingerprints. ML provided the laboratory supplies. All authors read and approved the final manuscript.

\section{Acknowledgements}

We thank Mr. Alfred Okeng for technical assistance. The Phoenix 100 ID/AST BDexpert system was purchased with previous support from the Swedish International Development Cooperation (Sida) and the Directorate of Research and Graduate Training, Makerere University. The Molecular Biology Laboratories of the Department of Medical Microbiology where the research was performed were previously supported by the National Institutes of Health (Grant Numbers RO3 Al062849 and RO1 Al075637).

\section{Competing interests}

The authors declare that they have no competing interests.

\section{Availability of data and materials}

The authors declare that all the data supporting the findings of this study are available within the article (and its Additional files).

\section{Ethics approval and consent to participate}

The isolates in this study were originally cultured from collected human specimens and hospital environment samples in previous studies $[8,18]$ that obtained Ethics Approval from the Makerere University School of Medicine Ethics Committee, and the Mulago Hospital Research and Ethics Committee. The Ethics Approval also covered the current study as the Written Informed Consent (or Assent for Minors) included consent for storage and use of stored samples/isolates in further studies. For human samples isolate data was delinked from patient data.

\section{Publisher's Note}

Springer Nature remains neutral with regard to jurisdictional claims in published maps and institutional affiliations.

Received: 20 August 2016 Accepted: 8 July 2017

Published online: 14 July 2017

\section{References}

1. Falagas ME, Bliziotis IA, Kasiakou SK, Samonis G, Athanassopoulou P, Michalopoulos A. Outcome of infections due to pandrug-resistant (PDR) Gram-negative bacteria. BMC Infect Dis. 2005;5:24.

2. Gootz TD, Marra A. Acinetobacter baumannii: an emerging multidrugresistant threat. Expert Rev Anti Infect Ther. 2008;6(3):309-25.

3. Strateva T, Yordanov D. Pseudomonas aeruginosa-a phenomenon of bacterial resistance. J Med Microbiol. 2009;58(Pt 9):1133-48.

4. Queenan AM, Bush K. Carbapenemases: the versatile beta-lactamases. Clin Microbiol Rev. 2007;20(3):440-58 (table of contents).

5. Manenzhe RI, Zar HJ, Nicol MP, Kaba M. The spread of carbapenemaseproducing bacteria in Africa: a systematic review. J Antimicrob Chemother. 2015;70(1):23-40.

6. Papp-Wallace KM, Endimiani A, Taracila MA, Bonomo RA. Carbapenems: past, present, and future. Antimicrob Agents Chemother. 2011;55(11):4943-60.

7. Perez F, Hujer AM, Hujer KM, Decker BK, Rather PN, Bonomo RA. Global challenge of multidrug-resistant Acinetobacter baumannii. Antimicrob Agents Chemother. 2007;51(10):3471-84. 
8. Kateete DP, Nakanjako R, Namugenyi J, Erume J, Joloba ML, Najjuka CF. Carbapenem resistant Pseudomonas aeruginosa and Acinetobacter baumannii at Mulago Hospital in Kampala, Uganda (2007-2009). SpringerPlus. 2016;5(1):1-11.

9. Najjuka CF, Kateete DP, Kajumbula HM, Joloba ML, Essack SY. Antimicrobial susceptibility profiles of Escherichia coli and Klebsiella pneumoniae isolated from outpatients in urban and rural districts of Uganda. BMC Res Notes. 2016;9:235

10. Okoche D, Asiimwe BB, Katabazi FA, Kato L, Najjuka CF. Prevalence and characterization of carbapenem-resistant Enterobacteriaceae isolated from Mulago National Referral Hospital, Uganda. PLoS ONE. 2015;10(8):e0135745.

11. Pitout JD, Revathi G, Chow BL, Kabera B, Kariuki S, Nordmann P, Poirel L. Metallo-beta-lactamase-producing Pseudomonas aeruginosa isolated from a large tertiary centre in Kenya. Clin Microbiol Infect Dis. 2008;14(8):755-9.

12. Lister PD, Wolter DJ, Hanson ND. Antibacterial-resistant Pseudomonas aeruginosa: clinical impact and complex regulation of chromosomally encoded resistance mechanisms. Clin Microbiol Rev. 2009;22(4):582-610.

13. Lee YC, Ahn BJ, Jin JS, Kim JU, Lee SH, do Song Y, Lee WK, Lee JC. Molecular characterization of Pseudomonas aeruginosa isolates resistant to all antimicrobial agents, but susceptible to colistin, in Daegu, Korea. J Microbiol. 2007;45(4):358-63.

14. Gu B, Tong M, Zhao W, Liu G, Ning M, Pan S. Prevalence and characterization of class I integrons among Pseudomonas aeruginosa and Acinetobacter baumannii isolates from patients in Nanjing, China. J Clin Microbiol. 2007:45(1):241-3.

15. Uma Karthika R, Srinivasa Rao R, Sahoo S, Shashikala P, Kanungo R, Jayachandran S, Prashanth K. Phenotypic and genotypic assays for detecting the prevalence of metallo-beta-lactamases in clinical isolates of Acinetobacter baumannii from a South Indian tertiary care hospital. J Med Microbiol. 2009;58(Pt 4):430-5

16. Manikal VM, Landman D, Saurina G, Oydna E, Lal H, Quale J. Endemic carbapenem-resistant Acinetobacter species in Brooklyn, New York: citywide prevalence, interinstitutional spread, and relation to antibiotic usage. Clin Infect Dis. 2000;31(1):101-6.

17. Mukonzo JK, Namuwenge PM, Okure G, Mwesige B, Namusisi OK, Mukanga D. Over-the-counter suboptimal dispensing of antibiotics in Uganda. J Multidiscip Healthc. 2013;6:303-10.

18. Seni J, Najjuka CF, Kateete DP, Makobore P, Joloba ML, Kajumbula H, Kapesa A, Bwanga F. Antimicrobial resistance in hospitalized surgical patients: a silently emerging public health concern in Uganda. BMC Res Notes. 2013;6:298

19. Labarca JA, Salles MJ, Seas C, Guzman-Blanco M. Carbapenem resistance in Pseudomonas aeruginosa and Acinetobacter baumannii in the nosocomial setting in Latin America. Crit Rev Microbiol. 2016;42(2):276-92.

20. Falagas ME, Vardakas $K Z$, Roussos NS. Trimethoprim/sulfamethoxazole for Acinetobacter spp.: a review of current microbiological and clinical evidence. Int J Antimicrob Agents. 2015;46(3):231-41.

21. SMI P 8: Laboratory detection and reporting of bacteria with carbapenem-hydrolysing beta-lactamases (carbapenemases). (https://www. gov.uk/government/publications/smi-p-8-laboratory-detection-andreporting-of-bacteria-with-carbapenem-hydrolysing-beta-lactamasescarbapenemases).

22. Inacio HSM, Bomfim MRQ, França RO, Farias LM, Carvalho MAR, Serufo JC, Santos SG. Phenotypic and genotypic diversity of multidrugresistant Pseudomonas aeruginosa isolates from bloodstream infections recovered in the Hospitals of Belo Horizonte, Brazil. Chemotherapy. 2014;60(1):54-62.

23. Pitondo-Silva A, Martins WV, Fernandes AFT, Stehling EG. High level of resistance to Aztreonam and Ticarcillin in Pseudomonas aeruginosa isolated from soil of different crops in Brazil. Sci Total Environ. 2014;473-474:155-8.

24. Santoro DO, Romao CM, Clementino MM. Decreased aztreonam susceptibility among Pseudomonas aeruginosa isolates from hospital effluent treatment system and clinical samples. Int J Environ Health Res. 2012;22(6):560-70.

25. Rawat D, Nair D. Extended-spectrum beta-lactamases in Gram negative bacteria. J Glob Infect Dis. 2010;2(3):263-74.

26. Thomson KS. Extended-spectrum-beta-lactamase, AmpC, and carbapenemase issues. J Clin Microbiol. 2010;48(4):1019-25.
27. Pirnay J-P, Bilocq F, Pot B, Cornelis P, Zizi M, Van Eldere J, Deschaght P, Vaneechoutte M, Jennes S, Pitt T, et al. Pseudomonas aeruginosa population structure revisited. PLoS ONE. 2009;4(11):e7740.

28. Salmon S, McLaws ML. Environmental challenges of identifying a patient zone and the healthcare zone in a crowded Vietnamese hospital. J Hosp Infect. 2015;91(1):45-52.

29. Sethi AK, Acher CW, Kirenga B, Mead S, Donskey CJ, Katamba A. Infection control knowledge, attitudes, and practices among healthcare workers at Mulago Hospital, Kampala, Uganda. Infect Control Hosp Epidemiol. 2012;33(9):917-23.

30. Versalovic J, Koeuth T, Lupski JR. Distribution of repetitive DNA sequences in eubacteria and application to fingerprinting of bacterial genomes. Nucleic Acids Res. 1991;19(24):6823-31.

31. Bhattacharya D, Sarma PM, Krishnan S, Mishra S, Lal B. Evaluation of genetic diversity among Pseudomonas citronellolis strains isolated from oily sludge-contaminated sites. Appl Environ Microbiol. 2003;69(3):1435-41

32. Dawson SL, Fry JC, Dancer BN. A comparative evaluation of five typing techniques for determining the diversity of fluorescent pseudomonads. J Microbiol Methods. 2002;50(1):9-22.

33. Hashemi A, Baghbani-Arani F. The effective differentiation of Salmonella isolates using four PCR-based typing methods. J Appl Microbiol. 2015;118(6):1530-40

34. Marques AS, Marchaison A, Gardan L, Samson R. BOX-PCR-based identification of bacterial species belonging to Pseudomonas syringae: $P$. viridiflava group. Genet Mol Biol. 2008;31(1):106-15.

35. Syrmis MW, O'Carroll MR, Sloots TP, Coulter C, Wainwright CE, Bell SC, Nissen MD. Rapid genotyping of Pseudomonas aeruginosa isolates harboured by adult and paediatric patients with cystic fibrosis using repetitive-element-based PCR assays. J Med Microbiol. 2004;53(Pt 11):1089-96.

36. Turton JF, Woodford N, Glover J, Yarde S, Kaufmann ME, Pitt TL. Identification of Acinetobacter baumannii by detection of the blaOXA51-like carbapenemase gene intrinsic to this species. J Clin Microbiol. 2006:44(8):2974-6.

37. Carroll KC, Glanz BD, Borek AP, Burger C, Bhally HS, Henciak S, Flayhart D. Evaluation of the BD Phoenix automated microbiology system for identification and antimicrobial susceptibility testing of Enterobacteriaceae. J Clin Microbiol. 2006;44(10):3506-9.

38. Miriagou V, Cornaglia G, Edelstein M, Galani I, Giske CG, Gniadkowski M, Malamou-Lada E, Martinez-Martinez L, Navarro F, Nordmann P, et al. Acquired carbapenemases in Gram-negative bacterial pathogens: detection and surveillance issues. Clin Microbiol Infect. 2010;16(2):112-22.

39. Asthana S, Mathur P, Tak V. Detection of carbapenemase production in Gram-negative bacteria. J Lab Physicians. 2014;6(2):69-75.

40. Broberg CA, Palacios M, Miller VL. Whole-genome draft sequences of three multidrug-resistant Klebsiella pneumoniae strains available from the American type culture collection. Genome Announc. 2013;1(3):e0031213. doi:10.1128/genomeA.00312-13.

41. Clinical, Institute LS. Performance standards for antimicrobic disc susceptibility tests: approved standard; M2-A9; 2006.

42. Andreou LV. Preparation of genomic DNA from bacteria. Methods Enzymol. 2013;529:143-51.

43. William S, Feil H. Bacterial genomic DNA isolation using CTAB. Sigma. 2012:50:6876.

44. Dombek PE, Johnson LK, Zimmerley ST, Sadowsky MJ. Use of repetitive DNA sequences and the PCR to differentiate Escherichia coli isolates from human and animal sources. Appl Environ Microbiol. 2000;66(6):2572-7.

45. Ateba CN, Mbewe M. Determination of the genetic similarities of fingerprints from Escherichia coli O157:H7 isolated from different sources in the North West Province, South Africa using ISR, BOXAIR and REP-PCR analysis. Microbiol Res. 2013;168(7):438-46.

46. Sachdeva P, Virdi JS. Repetitive elements sequence (REP/ERIC)-PCR based genotyping of clinical and environmental strains of Yersinia enterocolitica biotype $1 \mathrm{~A}$ reveal existence of limited number of clonal groups. FEMS Microbiol Lett. 2004:240(2):193-201.

47. Hunter PR. Reproducibility and indices of discriminatory power of microbial typing methods. J Clin Microbiol. 1990;28(9):1903-5.

48. Hunter PR, Gaston MA. Numerical index of the discriminatory ability of typing systems: an application of Simpson's index of diversity. J Clin Microbiol. 1988;26(11):2465-6. 